\title{
Gauge Choice in Witten's Energy Expression`
}

\author{
Thomas H. Parker
}

Department of Mathematics, Brandeis University, Waltham, MA 02154, USA

\begin{abstract}
Witten's equation $D \psi=0$ can be interpreted as a gauge fixing condition for classical supergravity. We rigorously prove the existence of asymptotically constant solutions of the more general gauge condition $D \psi=A \psi$ for almost all endomorphisms $A$ of the spin bundle. Each gives an expression for the gravitational energy similar to Witten's. These include the choice $A=\sqrt{\mathscr{R}}$, which yields the particularly elegant energy expression first noticed by Deser.
\end{abstract}

\section{Introduction}

Several years ago R. Schoen and S. T. Yau [12] succeeded in proving the Positive Energy Theorem in General Relativity. Shortly thereafter, E. Witten [14] discovered a second proof based on an integration-by-parts formula for the gravitational energy. These breakthroughs stimulated considerable recent work in relativity and have led to a much better understanding of the total mass and energy of isolated gravitational systems in General Relativity.

Witten's proof was to some extent inspired by supergravity, although supergravity plays no direct role in his argument. The exact manner in which Witten's energy expression emerges from supergravity has recently been clarified by Horowitz and Strominger, Deser, Teitelboim, and others (see [13] for references). In this context, the energy formula emerges from the underlying gauge theory of supergravity, and is seen to involve a gauge choice. Specifically, Witten used the Dirac equation $\not D \psi=0$ as a gauge-fixing condition. Many other choices are possible, and each yields an expression for the gravitational energy. These energy expressions display the gauge aspects of supergravity, yet do not involve the anticommuting fields nor supersymmetry which characterize the full supergravity theory.

This paper establishes several specific theorems about partial differential equations. These are intended to emphasize and clarify the gauge aspects of Witten's energy formula. Theorem 1 is a precise statement showing that an appropriate form of Witten's energy expression is valid even when Einstein's equations are not satisfied and when the spinor field does not satisfy the Dirac equation. Our main

^Partially supported by N. S. F. Grant MCS-82-02018 at Harvard University 
result (Theorem 2) is a P.D.E. existence theorem which allows one to make use of a very large class of gauge-fixing conditions. We give several applications, including the very natural gauge condition of Deser. The main results are stated in Sect. 2, and their rather technical proofs appear in Sect. 3.

The results of this paper simplify and generalize the analytic arguments of [10].

\section{The Main Results}

Let $M$ be an asymptotically flat spacelike hypersurface in a four-dimensional spacetime $N$. The Positive Energy Theorem asserts that the A.D.M. energymomentum $\left(E \cdot p_{k}\right)$ of $M-$ which is defined as an integral over the 2-sphere at infinity in $M$-is positive in the sense that $E-|p| \geqq 0$. Witten's proof of this theorem $([14,10])$ involves considering the "hypersurface Dirac operator $D$;" this is the composition

$$
\Gamma(S) \stackrel{\nabla}{\rightarrow} \Gamma\left(T^{*} M \oplus S\right) \stackrel{c}{\rightarrow} \Gamma(S),
$$

where $S=S_{+} \otimes S_{-}$is the (four component) spin bundle of $N$ along $M, \nabla$ is the metric connection of $N$ and $c$ is Clifford multiplication. (If we choose a local orthonormal basis of 1 -forms of $M$ and let $\left\{\gamma^{i}\right\}$ be the corresponding $\gamma$-matrices, then locally

$$
D \psi=\sum_{(i=1)}^{3} \gamma^{i} \nabla_{i} \psi
$$

for $\psi \in \Gamma(S)$.) The key ingredient of Witten's proof is an integration by parts formula which can be cast in the following general form:

Theorem 1. Let $M$ be an asymptotically flat (as defined in [10]) spacelike hypersurface in a four-dimensional Lorentz manifold $N$ whose Riccicurvature along $M$ satisfies $R_{i j} \in L^{1}(M)$. Let $\psi$ be any spinor $\psi \in \Gamma(S)$ which approaches a constant spinor $\psi_{0}$ at infinity at a rate such that

(i) $\left|\nabla\left(\psi-\psi_{0}\right)\right| \in L^{2}$,

(ii) $\sigma^{-1}\left(\psi-\psi_{0}\right) \in L^{2}$ for some bounded function $\sigma$ with $\sigma=|x|$ on the asymptotic ends.

Then

$$
4 \pi G\left\langle\psi_{0},\left(E-p_{k} \gamma^{0} \gamma^{k}\right) \psi_{0}\right\rangle=\int_{M}|\nabla \Psi|^{2}+\langle\psi, \mathscr{R} \psi\rangle-|\not D \psi|^{2} d v_{g}
$$

Here $G$ is the gravitational constant, $\gamma^{0}$ is the $\gamma$ matrix of the 1 -form normal to $T^{*} M$ and $d v_{g}$ is the Riemannian volume form of $M$. The inner product $\langle\psi, \phi\rangle$ is the positive definite hermitian inner product described in [10] and usually denoted $\psi^{\dagger} \phi$ by physicists. Finally, $\mathscr{R}=\frac{1}{4}\left(R+2 R_{00}+2 R_{0 i} \gamma^{0} \gamma^{i}\right)$ is an endomorphism of $S$ constructed from the scalar curvature $R$ and the Ricci tensor $R_{i j}$ of $N$.

Theorem 1 was essentially proved in [10]; it follows from a careful examination of the proof of Theorem 4.1 (iii) of that paper. It should be emphasized that Theorem 
1 does not require Einstein's equations, and holds for any spinor satisfying (i) and (ii): $\psi$ need not satisfy any equation.

We note in passing that if $N$ satisfies Einstein's equations and the Dominant Energy Condition then $\mathscr{R}$ is non-negative. We can then use the Monotonerather than the Dominated-Convergence Theorem to prove Theorem 1 without the hypothesis that $R_{i j} \in L^{1}$.

Witten's argument applies (1) by finding asymptotically constant spinors with $D \psi=0$; the righthand side of (1) is then manifestly non-negative and the Positive Energy Theorem follows by taking $\psi_{0}$ to be the eigenvector of $p_{k} \gamma^{0} \gamma^{k}$ with eigenvalue $|p|$. Now in the context of supergravity (cf. $[3,13])$ the equation

$$
\not \psi=0
$$

should be regarded as a gauge-fixing condition which provides a canonical way of extending $\psi_{0}$ across $M$. It is then natural to consider alternative gauge conditions of the form

$$
\not \psi=A \psi,
$$

where $A$ is any endomorphism of the spin bundle $S$. The main purpose of this note is to establish the existence of asymptotically constant solutions of (3). We will do this for large collections of endomorphisms $A$ - the "admissible families" of Definition 3.6. For example, one such admissible family is the set of all $C^{1}$ endomorphisms $A$ with $|A| \in L^{2}$ and $A=O\left(r^{-3 / 2}\right)$ at infinity. Our main result-proven later-asserts the existence of solutions of (3) for almost all $A$ in such a family:

Theorem 2. Let $M$ be an asymptotically flat spacelike hypersurface in a spacetime $N$ and suppose that Einstein's equations and the dominant energy condition hold along $M$. Let $\mathscr{B}$ be an admissible family of endomorphisms in the sense of Definition 3.6 below. Then for each $A$ in a open dense set $\mathscr{I}$ of $\mathscr{B}$, and for each constant spinor $\psi_{0}$, there exists a unique spinor $\psi \in \Gamma(S)$ satisfying conditions (i) and (ii) of Theorem 1 and

$$
D_{A} \psi=(D+A) \psi=0 \text {. }
$$

Our proofs of this and the next theorem will also give precise statements about the uniform decay of $\psi$ at infinity (Eq. 17 below).

Remarks. (1) In a completely analogous way, we could consider the Dirac operators $D_{g}$ associated to different asymptotically flat metrics $g$ for $N$ along $M$, and show that one can solve $D_{g} \psi=0$ for almost all such $g$, and in particular for all $g$ whose curvature endomorphism $\mathscr{R}$ is non-negative.

(2) The fact that (4) can be solved for almost all-but not all $-A$ is a typical feature of gauge theories. The exceptional values of $A$ are analogous to the reducible connections in Yang-Mills theory, and to the metrics with Killing fields in classical relativity (regarded as a gauge theory with the diffeomorphism group as the gauge group).

For each endomorphism $A$ Eq. (4) breaks the gauge freedom in the general energy expression (1) by specifying $\psi$. In particular it is clear that $\psi$, being a gauge dependent field, has no physical meaning. 
Deser ([13]) has pointed out that there is a particularly natural choice for the endomorphism $A$. Because $\mathscr{R}$ is a non-negative endomorphism, $\sqrt{\mathscr{R}}$ exists. Taking $A=t \sqrt{\mathscr{R}}, t \in \mathbb{R}$, we can then prove:

Theorem 3. Let $M$ be as in Theorem 2, and assume that the stress-energy tensor satisfies $T_{i j} \in L^{1}(\mathrm{M})$. Then for each constant spinor $\psi_{0}$, and for each $t \in[-1-\delta$, $1+\delta$ ] for some $\delta>0$, there is a $C^{\infty}$ spinor $\psi$ satisfying conditions (i), (ii) of Theorem 1 and

$$
D \psi=t \sqrt{\mathscr{R}} \psi .
$$

Hence when $\psi_{0}$ is an eigenvector of $p_{k} \gamma^{0} \gamma^{k}$ with eigenvalue $|p|$ and $\left|\psi_{0}\right|^{2}=1$, (1) gives

$$
E-|p|=(4 \pi G)^{-1} \int_{M}|\nabla \psi|^{2}+\left(1+t^{2}\right)\langle\psi, \mathscr{R} \psi\rangle d v_{g} .
$$

When $t=0$ this is Witten's energy expression. However, for any $t \in[-1,1]$ the righthand side is manifestly non-negative, so $E-|p| \geqq 0$, and (as in [10]) equality holds if and only if $M=\mathbb{R}^{3}$ with the standard metric. In particular, the choice $t=1$, corresponding to Deser's gauge condition

$$
\not D \psi=\sqrt{\mathscr{R}} \psi,
$$

is possible, and yields a proof of the Positive Energy Theorem and an energy expression

$$
E-|p|=(4 \pi G)^{-1} \int_{M}|\nabla \psi|^{2} d v_{g}
$$

every bit as natural and elegant as Witten's original choice.

Equation (6) clearly shows that the two terms in Witten's energy expression (the case $t=0$ ) cannot be interpreted as the energies of the gravitational and matter fields respectively; the separation of the two terms is purely an artifact of the gauge choice (2). (Horowitz and Strominger [5] have previously shown that in the Newtonian limit these two terms do not reduce to the usual expressions for the Newtonian gravitational and rest mass energies.)

Theorem 2 has other applications. For example, Moreshi and Sparling [8], following Gibbons, Hawking, Horowitz, and Perry [4], give a version of Witten's energy expression which includes electromagnetic charges. Their derivation assumes the existence of a certain P.D.E. (Eq. 3.6 of [8]). Under the hypotheses of their main theorem this existence follows from Theorem 2.

\section{Proofs of the Main Theorems}

We shall prove Theorem 2 using weighted Sobolev spaces. Specifically, we will work with the spaces $H_{k, \delta}^{p}$, obtained by completing the space of smooth compactly supported sections of $S$ in the norm

$$
|\psi|_{p, k, \delta}=\sum_{l=0}^{k}\left|\sigma^{\delta+l} \bar{\nabla}^{l} \psi\right|_{p},
$$


where ||$_{p}$ is the $L^{p}$ norm on $M, \sigma$ is a fixed smooth function with $\sigma \geqq 1$ and $\sigma(x)=|x|$ for $x$ in the asymptotic ends of $M$, and $\bar{\nabla}^{l}$ is the $l$-fold composition $\bar{\nabla} \ldots \bar{\nabla}$ of the Riemannian spin connection of $M$. (It is appropriate to use the connection $\bar{\nabla}$ in (9) because it is compatible with the inner product $\langle$,$\rangle on S$, whereas $\nabla$ is not.)

A useful observation, proven in the appendix, is that the norm (9) is equivalent to its top order term, that is

$$
|\psi|_{p, k, \delta} \leqq c_{1}\left|\sigma^{\delta+k} \bar{\nabla}^{k} \psi\right|_{p}
$$

for some constant $c_{1}$. Now the metric connections of $M$ and $N$ are related by $\bar{\nabla}_{i}=\nabla_{i}+\frac{1}{2} h_{i j} \gamma^{0} \gamma^{j}$, where $h_{i j}$ is the second fundamental form of $M \subset N$, and $\left|h_{i j}\right| \leqq$ $c_{2} \sigma^{-1}$ as a consequence of asymptotic flatness. Hence (10) gives

$$
|\psi|_{p, 1, \delta} \leqq c_{3}\left|\sigma^{1+\delta} \nabla \psi\right|_{p} .
$$

The main reason for introducing the spaces $H_{k, \delta}^{p}$ for $p \neq 2$ is that when $p>1$ and $3<p k$ the elements $\psi \in H_{k, \delta}^{p}$ have uniform decay: for each $\alpha<\delta+3 / p$ there is a constant $c$ such that $|\psi| \leqq c \sigma^{-\alpha}$ pointwise (cf. Cantor [2]). One consequence of this is that

$$
H_{k, \delta}^{p} \subset H_{1,-1}^{2}
$$

whenever $k>1+3 / p$ and $\delta>\frac{1}{2}-3 / p$.

The theory of elliptic operators on these weighted spaces was initiated by Nirenberg and Walker, and has been developed in recent years by M. Cantor, Choquet-Bruhat and Christodoulou, R. Lockhart, and R. McOwen (see [2] for references). Using their results we shall prove:

Theorem 4. Suppose $A \in \operatorname{End}(S)$ is measurable and $\left|\sigma^{1+\varepsilon} A\right|$ is bounded for some $\varepsilon>0$. Then for $-3 / p<\delta<2-3 / p$,

$$
D_{A}=\not+A: H_{1, \delta}^{p} \rightarrow H_{0, \delta+1}^{p}
$$

is Fredholm and there is a constant c such that $\forall \psi \in H_{k, \delta}^{p}$,

$$
|\psi|_{p, k, \delta} \leqq c\left(\left|D_{A} \psi\right|_{p, k-1, \delta+1}+|\psi|_{p, k-1, \delta+1}\right) .
$$

For $p \geqq 2$ and $\frac{1}{2}-3 / p \leqq \delta<\frac{3}{2}-3 / p, D_{A}$ has index 0 and (13) is an isomorphism whenever $D_{A}: H_{1,-1}^{2} \rightarrow L^{2}$ is injective.

Proof. Standard results $([2,7])$ imply that $\not D: H_{1, \delta}^{p} \rightarrow H_{0, \delta+1}^{p}$ is Fredholm for $-3 / p$ $<\delta<2-3 / p$, and that (14) holds for $\not D$. If $\sigma^{1+\varepsilon} A$ is bounded and measurable for some $\varepsilon>0$ then $A: H_{1, \delta}^{p} \rightarrow H_{0, \delta+1}^{p}$ is a compact operator ([7] Theorem 5.2). Hence by general Fredholm theory $([6]) \not D+A$ is Fredholm with index $D_{A}=$ index $D$. Equation (14) then follows immediately from the corresponding inequality for $D$.

For $p \geqq 2$ and $\delta \geqq \frac{1}{2}-3 / p$ we have $H_{1, \delta}^{p} \subset H_{1,-1}^{2}$ by Holder's inequality. Thus the injectivity of $D_{A}: H_{1,-1}^{2} \rightarrow L^{2}$ implies that (13) is injective. To prove that it is an isomorphism we need only show that $D$-and therefore $D_{A}$ - has index 0 .

Lemma 5 below shows that $D$ is injective on $H_{1,-1}^{2}$, and hence on $H_{1, \delta}^{p}$. To establish surjectivity, suppose that $\psi$ is in the kernel of the dual map $\mathbb{D}^{*}: H_{0,-\delta-1}^{q} \rightarrow$ $\left(H_{1, \delta}^{p}\right)^{*}, q^{-1}=1-p^{-1}$. Then $D \psi=0$ weakly because $\not D$ is formally self-adjoint (Eq. 3.6 of [10]). Standard elliptic estimates imply that $\psi$ is smooth. Let $\beta_{R}$ be a 
smooth cutoff function with support in $\mathbb{R}^{n}-B(R)$, with $\beta_{R}=1$ on $\mathbb{R}^{n}-B(2 R)$, and $\left|d \beta_{R}\right| \leqq c \sigma^{-1}$ pointwise. Repeatedly applying (14) to $\left(1-\beta_{R}\right) \psi$ and letting $R \rightarrow \infty$ shows that $\psi \in H_{k,-\delta-1}^{p} \forall k$. But when $\delta<\frac{3}{2}-3 / p$, we then have $\psi \in H_{1,-1}^{2}$ by (12), and hence $\psi=0$ by Lemma 5. Thus $\mathbb{D}: H_{1, \delta}^{p} \rightarrow H_{0, \delta+1}^{p}$ has index 0 .

Lemma 5. There are constants $c, \delta>0$ such that $\forall t \in[-1-\delta, 1+\delta]$ and $\psi \in H_{1,-1}^{2}$,

$$
|\psi|_{2,1,-1} \leqq c_{4}|(\not D+t \sqrt{\mathscr{R}}) \psi|_{2} .
$$

Proof. By Theorem 1 with $\psi_{0}=0$ we have

$$
|\not \nabla|_{2}^{2}=|\nabla \psi|_{2}^{2}+\langle\psi \mathscr{R} \psi\rangle,
$$

$\forall \psi \in C_{c}^{\infty}$. But $R$ is continuous and $O\left(r^{-3}\right)$ by hypothesis, so $|\sqrt{\mathscr{R}}| \leqq c_{5} \sigma^{-1}$. Taking limits establishes (15) $\forall \psi \in H_{1,-1}^{2}$. The inequality $2 a b \leqq(1+\varepsilon)^{-1} a^{2}+$ $(1+\varepsilon) b^{2}$ then gives, for each $\varepsilon>0$,

$$
\begin{aligned}
|(\not D+t \sqrt{\mathscr{R}}) \psi|_{2}^{2} & \geqq|D \psi|_{2}^{2}-2|\not D \psi|_{2}|t \sqrt{\mathscr{R}} \psi|_{2}+|t \sqrt{\mathscr{R}} \psi|_{2}^{2} \\
& \geqq\left[1-(1+\varepsilon)^{-1}\right]\left(|\nabla \psi|_{2}^{2}+|\sqrt{\mathscr{R}} \psi|_{2}^{2}\right)-e t^{2}|\sqrt{\mathscr{R}} \psi|_{2}^{2} \\
& =\frac{\varepsilon}{1+\varepsilon}\left(|\nabla \psi|_{2}^{2}-\varepsilon_{t}|\sqrt{\mathscr{R}} \psi|_{2}^{2}\right),
\end{aligned}
$$

where $\varepsilon_{t}=(1+\varepsilon) t^{2}-1$. Using (11) and $|\sqrt{\mathscr{R}} \psi|_{2} \leqq c_{5}|\psi|_{2,1,-1}$, we have

$$
|(\not D+t \sqrt{\mathscr{R}}) \psi|_{2}^{2} \geqq \varepsilon(1+\varepsilon)^{-1}\left(c_{3}-\varepsilon_{t} c_{5}^{2}\right)|\psi|_{2,1,-1}^{2} .
$$

Now take $\varepsilon=c_{3} / 4 c_{5}^{2}$ and $\delta$ small enough that $\varepsilon_{t} \leqq c_{3} / 2 c_{5}^{2} \forall t \in[-1-\delta, 1+\delta]$.

Together, the last two results show that (13) is an isomorphism for at least some $A$. We are going to show that this is the case for almost all $A$. To this end we introduce some spaces of endomorphisms $A$.

Definition 6. (i) For each $\varepsilon>0$ set

$$
A_{\varepsilon}=\left\{A \in \operatorname{End}(S) \mid A \in H_{0,1+\varepsilon}^{\infty} \cap L^{2}\right\} .
$$

Each $A \in A_{\varepsilon}$ is a bounded linear operator $A: H_{1,-1-\varepsilon}^{2} \rightarrow L^{2}$; the operator norm makes $A_{\varepsilon}$ a Banach space and $A_{\varepsilon} \subseteq A_{0}$ continuously.

(ii) A linear subspace $\mathscr{B} \subset A_{\varepsilon}$ is admissible if for each $B \in \mathscr{B}$ for which $D_{B}: H_{1,-1}^{2} \rightarrow L^{2}$ has a non-zero kernel there exist $L \in \mathscr{B}, \psi \in \operatorname{ker} \emptyset_{B}$ and $\phi \in \operatorname{ker} D_{B}^{+}$ such that

$$
\int_{M}\langle\phi, L \psi\rangle \neq 0
$$

This criterion for admissibility is very weak; it would appear that almost any choice of $L$ should satisfy (16). Nevertheless to prove (16) in specific cases is not entirely trivial, and becomes increasingly more difficult as $\mathscr{B}$ is made smaller. As an example of such a proof we have:

Lemma 7. Let $A_{\varepsilon}$ be a family of differentiable endomorphisms which contains the set $C_{c}^{\infty}(S, S)$ of smooth compactly supported endomorphisms. Then $\mathscr{B}$ is admissible.

Proof. Suppose that $D_{B}$ has a non-zero kernel. Then the adjoint $D_{B}^{\dagger}$ also has a non- 
zero kernel because $D_{B}$ has index 0 . Fix $\psi \in \operatorname{ker} D_{B}$ and $\phi \in \operatorname{ker} D_{B}^{\dagger}$ Standard elliptic estimates ([9], Theorem 6.4.3) imply that $\psi$ and $\phi$ are continuous and lie in the Sobolev space $H_{2, \text { loc }}^{2}$. The zero set $Z(\psi)=\{x \mid \psi(x)=0\}$ is then closed, and by the unique continuation theorem of Aronszajn ([1]) it has no interior. Thus the complement $Z^{c}(\psi)$ (and similarly $Z^{c}(\phi)$ ) is open and dense. Choose a smooth positive function $\zeta(x)$ with compact support in $Z^{c}(\psi)$ and set $L(\xi)=\langle\zeta, \psi\rangle \phi$. Then

$$
\left.\int_{M}\langle\phi, L \psi\rangle=\int_{M} \zeta|\psi|^{2}|\phi|^{2}\right\rangle 0 .
$$

Finally, if $L$ is not smooth we can replace it by an $L^{\prime} \in C_{c}^{\infty}(S, S)$ which is close enough to $L$ that (16) still holds.

Proposition 8. If $\mathscr{B}$ is an admissible family then $\mathscr{I}=\left\{B \in \mid D_{B}\right.$ is invertible $\}$ is open and dense in $\mathscr{B}$.

Proof. For each $B \in \mathscr{B} \subset A_{\varepsilon}, B: H_{1,-1}^{2} \rightarrow L^{2}$ is compact, $D^{-1} D_{B}=1+D^{-1} B$ is Fredholm, and

$$
P_{B}=\left(\not^{-1} \not D_{B}\right)^{\dagger}\left(D^{-1} D_{B}\right)=\not_{B}^{\dagger} \not D^{-2} D_{B}
$$

is an analytic family of self-adjoint Fredholm operators on $H_{1,-1}^{2}$. Each $P_{B}$ gives a spectral decomposition of $H_{1,-1}^{2}$, and the spectrum and the eigenfunctions depend analytically on $B\left([11]\right.$ Theorem XII.13). In particular, dimker $D_{B}$ is upper semicontinuous and $\mathscr{I}=\left\{B \in \mathscr{B} / P_{B}\right.$ has no zero eigenvalue $\}$ is open in $\mathscr{B}$.

To show that $\mathscr{I}$ is dense we fix $B \notin \mathscr{I}$ and $\psi, \phi$ and $L$ as in (16), and consider the perturbations $P_{t}=P_{B+t L}$ of $P_{B}$. There are then $L^{2}$ orthonormal eigenfunctions $\psi_{i}(t)$ and numbers $\lambda_{i}(t), i=1, \ldots, N=\operatorname{dim} \operatorname{ker} D_{B}$, which depend analytically on $t$ for $|t|$ small such that (a) $\lambda_{i}(0)=0$, (b) the $\psi_{i}(0)$ are an $L^{2}$ orthonormal basis of ker $D_{B}$ with $\psi_{1}(0)=\psi$, and (c) $P_{t} \psi_{i}(t)=\lambda_{i}(t) \psi_{i}(t)$. Taking the $L^{2}$ inner product with $\psi_{i}(t)$ yields

$$
\lambda_{i}(t)=\left\langle\psi_{i}(t), P_{t} \psi_{i}(t)\right\rangle \text {. }
$$

Differentiating,

$$
\lambda^{\prime}(t)=\langle\psi, P \psi\rangle+\left\langle\psi, P^{\prime} \psi\right\rangle+\langle P \psi, \psi\rangle .
$$

At $t=0$ we have $P_{0} \psi=0, P_{0}^{\prime}=L^{\dagger} \not D^{-2} D_{0}+D_{0}^{\dagger} D_{L}^{-1}$, and $D_{0} \psi=0$, so $\lambda^{\prime}(0)=0$. Differentiating again,

$$
\frac{1}{2} \hat{\lambda}^{\prime \prime}(0)=\left\langle\psi^{\prime}, P^{\prime} \psi\right\rangle+\left\langle\psi^{\prime}, P \psi^{\prime}\right\rangle+\frac{1}{2}\left\langle\psi, P^{\prime \prime} \psi\right\rangle+\left\langle\psi, P^{\prime} \psi^{\prime}\right\rangle .
$$

Since $D$ is self-adjoint and $P^{\prime \prime}(0)=2 L^{\dagger} \not D^{-2} L$,

$$
\frac{1}{2} \lambda_{1}^{\prime \prime}(0)=\left|\not D^{-1}\left(D_{0} \psi+L \psi\right)\right|^{2} .
$$

Thus $\lambda_{1}^{\prime \prime}(0) \geqq 0$ with equality if and only if $D_{0} \psi=-L \psi$. But then

$$
0=\left\langle D_{0}^{\dagger} \phi, \psi\right\rangle=\left\langle\phi, D_{0} \psi\right\rangle=\langle\phi, L \psi\rangle,
$$

contradicting (16). Thus $\lambda_{1}^{\prime \prime}(0)>0$ and $\operatorname{dim} \operatorname{ker} \emptyset_{t} \leqq \operatorname{dim} \operatorname{ker} \emptyset_{0}-1$ for all sufficiently small $t$. Repeating this process a finite number of times yields invertible endomorphisms $B^{\prime} \in \mathscr{I}$ arbitrarily close to $B$. 
Proof of Theorem 2. Fix $A \in \mathscr{I} \subset \mathscr{B}$ as in Proposition 8. Let $\psi_{1}$ be a spinor such that $\psi_{1}(x)=\psi_{0}(x)$ for all $x$ in the asymptotic end with $|x|>$ some $R$. The conditions of asymptotic flatness (cf. [8]) insure that $D \psi_{1}=O\left(r^{-2}\right)$, and $A \in L^{2}$ by Definition 6. Hence $D_{A} \psi_{1} \in L^{2}$. Now $\not_{A}: H_{1,-1}^{2} \rightarrow L^{2}$ is injective by Proposition 8 , so by Theorem 4

$$
\not D_{A}: H_{1, \delta}^{p} \rightarrow H_{0,1+\delta}^{p}
$$

is an isomorphism for $p \geqq 2$ and $\frac{1}{2}-3 / p \leqq \delta<\frac{3}{2}-3 / p$. Hence there is a unique $\xi \in H_{1,-1}^{2}$ such that $D_{A} \xi=D_{A} \psi_{1}$. The spinor $\psi=\psi_{1}-\xi$ then satisfies conditions (i) and (ii) of Theorem 1 , and $D_{A} \psi=0$.

If we assume a stronger decay on $A$ we obtain a uniform decay rate for $\psi$. Specifically, if $A \in A_{\varepsilon}$ for some $\varepsilon>\frac{1}{2}$ (i.e. $A=O\left(r^{-1-\varepsilon}\right)$ ), then $D_{A} \psi_{1} \in H_{0,1+\delta}^{p}$ for $\frac{1}{2} \leqq \delta+3 / p<\varepsilon \leqq 1$. Inverting $D_{A}$ on $H_{0,1+\delta}^{p}$ as above and using the facts mentioned before (12), we find that

$$
|\psi| \leqq c \sigma^{-\alpha}
$$

for some constant $c$ and $\alpha<\varepsilon \leqq 1$.

Proof of Theorem 3. We have $A=t \sqrt{\mathscr{R}} \gamma^{0} \in L^{2}$, since $T_{i j} \in L^{1}$, and $A=O\left(r^{-3 / 2}\right)$ by asymptotic flatness. For $t \in[-1-\delta, 1+\delta]$ Lemma 5 shows that $\not D+$ $t \sqrt{\mathscr{R}}: H_{1,-1}^{2} \rightarrow L^{2}$ is injective. The theorem follows as in the preceding proof.

If $\left|T_{i j}\right|=O\left(r^{-2-2 \varepsilon}\right)$ for some $\frac{1}{2}<\varepsilon \leqq 1$, we again obtain the uniform decay (17). In particular in many physically interesting situations (e.g. electromagnetic fields with compactly supported sources) we have $T_{i j}=O\left(r^{-4}\right)$, and hence $\psi=O\left(r^{-\alpha}\right) \forall \alpha<1$.

\section{Appendix}

The inequality (10) is an elementary fact about weighted Sobolev spaces which unfortunately does not appear in the literature. We will give a general proof here.

Let $M$ be an $n$-dimensional Riemannian manifold that is asymptotically flat in the following weak sense. There is a compact set $K \subset M$ such that $M-K$ is the disjoint union of a finite number of asymptotic ends $M_{l}$, each diffeomorphic to the complement of a ball in $\mathbb{R}^{n}$. Under this diffeomorphism the metric on $M_{l}$, written in standard coordinates on $\mathbb{R}^{n}$, should satisfy $\left|g_{i j}-\delta_{i j}\right| \rightarrow 0$ as $r \rightarrow \infty$. Let $E$ be a hermitian vector bundle over $M$ with connection $\nabla$ compatible with the metric. We then have

Proposition. For $\delta>-n / p$, there is a constant $c$ such that

$$
|\phi|_{p, k, \delta} \leqq c\left|\alpha^{k+\delta} \nabla^{k} \phi\right|_{p} . \quad \forall \phi \in H_{k, \delta}^{p}(E) .
$$

Note that if $\delta<-n / p$ and $E$ is a trivial bundle then $H_{k, \delta}^{p}$ contains the constant functions and the inequality fails.

Proof. By continuity it suffices to verify (17) for smooth compactly supported sections $\phi$. For notational simplicity we shall assume that $M$ has only one end, so $M-K=\mathbb{R}^{n}-B$ for some ball $B=B(R)$. Since $\sigma$ is bounded above and below on 
$K$, a standard estimate gives

$$
\begin{aligned}
\int_{K}\left|\sigma^{\delta} \phi\right|^{p} & \leqq c_{1} \int_{K}|\phi|^{p} \leqq c_{2} \int_{K}|\nabla \phi|^{p}+c_{2} \int_{S}|\phi|^{p} \\
& \leqq c_{3} \int_{K}\left|\sigma^{1+\delta} \nabla \phi\right|^{p}+c_{4} \int_{K} \sigma^{\delta}|\phi|^{p},
\end{aligned}
$$

where $S=\partial K$ (see Theorem 3.6 .4 of [9] - the proof given there applies on manifolds).

On the end $B^{c}=\mathbb{R}^{n}-B$ we have $\sigma=r$ and the Riemannian volume form $d v_{g}$ is related to the Euclidean volume form (in spherical coordinates) by

Hence

$$
c_{5}^{-1} d v_{g} \leqq \sigma^{n-1} d r d \Omega \leqq c_{5} d v_{g} .
$$

$$
\int_{B^{c}}\left|\sigma^{\delta} \phi\right|^{p} d v_{g} \leqq c_{6} \int_{B^{c}}|\phi|^{p} d\left(\sigma^{p \delta+n}\right) d \Omega,
$$

where $c_{6}=c_{1}(p \delta+n)^{-1}>0$. By Stokes' theorem this is

$$
=-c_{6} \int_{S} \sigma\left|\sigma^{\delta} \phi\right|^{p} \cdot \sigma^{n-1} d \Omega-c_{6} \int_{B^{c}} d\left(|\phi|^{p}\right) \cdot \sigma^{p \delta+n} d \Omega .
$$

But $\left|d\left(|\phi|^{p}\right)\right|=p / 2|\phi|^{p-2}|\langle\nabla \phi, \phi\rangle+\langle\phi, \nabla \phi\rangle| \leqq p|\phi|^{p-1}|\nabla \phi|$, so the above is

$$
\leqq-c_{6} c_{5}{ }^{-1} \int_{S} \sigma\left|\sigma^{\delta \phi}\right|^{p}+p c_{6} c_{5} \int_{B^{c}}\left|\sigma^{\delta} \phi\right|^{(p-1)} \cdot\left|\sigma^{1+\delta} \nabla \phi\right| d v_{g} .
$$

For any $\varepsilon>0$ we can now apply Young's inequality $a b \leqq \varepsilon a^{q}+\varepsilon^{1-p} b^{p}$, where $p^{-1}+q^{-1}=1$, to obtain

$$
\int_{B^{c}}\left|\sigma^{\delta} \phi\right|^{p} d v_{g} \leqq-c_{6} c_{5}^{-1} \int_{s} \sigma\left|\sigma^{\delta \phi}\right|^{p}+c_{7} \varepsilon \int_{B^{c}}\left|\sigma^{\delta} \phi\right|^{p} d v_{g}+c_{7} \varepsilon^{1-p}|\bar{\nabla} \phi|_{p, 0, \delta+1}^{p} .
$$

Taking $\varepsilon=\frac{1}{2} c_{7}^{-1}$ and subtracting,

$$
\int_{B^{c}}\left|\sigma^{\delta} \phi\right|^{p} d v_{g} \leqq-c_{8} \int_{S} \sigma\left|\sigma^{\delta} \phi\right|^{p}+c_{9}|\nabla \phi|_{p, 0, \delta+1}^{p} .
$$

Combining (18) and (19) gives

$$
|\phi|_{p, 1, \delta} \leqq|\nabla \phi|_{p, 0, \delta+1}+|\phi|_{p, 0, \delta} \leqq c|\nabla \phi|_{p, 0, \delta+1} .
$$

This completes the proof of the case $k=1$.

Now suppose that (17) holds for some $k$. Replacing $E$ by $E \otimes\left(\otimes{ }^{k} T . M\right)$ and $\phi$ by $\nabla^{k} \phi$ in (20) then gives

$$
|\phi|_{p, k, \delta} \leqq c_{k}\left|\nabla^{k} \phi\right|_{p, 0, \delta+k} \leqq c_{k}^{\prime}\left|\nabla^{k+1} \phi\right|_{p, 0 \delta+k+1} .
$$

Hence

$$
|\phi|_{p, k+1, \delta}=\left(1+c_{k}^{\prime}\right)\left|\nabla^{k+1} \phi\right|_{p, 0, \delta+k+1},
$$

and the proposition follows by induction.

Remark. The tensor $\nabla^{k} \phi=\nabla \circ \cdots \circ \nabla \phi$ is not symmetric in its indices; it differs from 
its symmetrization $\nabla_{s}^{k} \phi$ by an operator $A$ of the form

$$
A=\nabla^{k}-\nabla_{s}^{k}=\sum_{|\alpha|=0}^{k-2} a_{m} \nabla^{\alpha_{1}} R \cdot \nabla^{\alpha_{2}} R \cdots \nabla^{k-|\alpha|-2},
$$

where $|\alpha|=\sum \alpha_{i}+2$ and $R$ represents the curvatures of $M$ and $E$. The norms (9) based on $\nabla^{k}$ and $\nabla_{s}^{k}$ are equivalent if $\nabla^{l} R=O\left(r^{-2-t}\right)$ for $0 \leqq l \leqq k-2$.

\section{References}

1. Aronszajn, N.: A unique continuation theorem for solutions of elliptic partial equations. J. Math. Pure App. 36, 235-249 (1957)

2. Cantor, M.: Elliptic operators and the decomposition of tensor fields. AMS Bul. Vol 5 No 3, 235-262, (1981)

3. Deser, S.: Supergauge - independence of Witten's gravitational energy expression. Phys. Rev D15 (to appear)

4. Gibbons, G. W., Hawking, S., Horowitz, G., Perry M.: Positive mass theorems for black holes. Commun. Math. Phys. 88, 295-308 (1983)

5. Horowitz, G., Strominger A.: On Witten's extression for gravitational energy. Phys. Rev. D27, 2793 (1983)

6. Lang, S.: Real analysis. Reading, MA.: Addison-Wesley, 1983

7. Lockhart, R.: Freedholm properties of a class of elliptic operators on non-compact manifolds. Duke J. 48, 289-312 (1981)

8. Moreschi, O., Sparling A.: On the positive energy theorem involving mass and electromagnetic charges. Commun. Math. Phys. 95, 113-120 (1984)

9. Morrey, C. B.: Multiple integrals in the calculus of variations. Berlin, Heidelberg, New York: Springer 1966.

10. Parker, T., Taubes, C.: On Witten's proof of the positive energy theorem. Commun. Math. Phys. 84, 223-238 (1982)

11. Reed, M.: Simon, B.: Methods of modern mathematical analysis, Vol 4. New York: Academic Press, 1978

12. Schoen, R., Yau, S. T.: On the proof of the positive mass conjecture in general relativity. Commun. Math. Phys. 65, 45-76 (1976); Proof of the positive mass theorem II. Commun. Math. Phys. 79, 231 (1981)

13. Teitelboim, C.: Manifestly positive-energy expression in classical gravity: simplified derivation from supergravity. Phys. Rev D29, 2763 (1984)

14. Witten, E.: A new proof of the positive energy theorem. Commun. Math. Phys. 80, 381-402 (1981)

Communicated by A. Jaffe

Received October 4, 1984; in revised form January 11, 1985 